${ }^{1}$ Centro de Salud Familiar María Latife Saadi, Corporación Municipal de Rancagua, Chile Universidad Católica del Maule. ${ }^{a}$ Magister en Ciencias Médicas. bEnfermera Universitaria.

Trabajo no recibió financiamiento. Los autores declaran no tener conflictos de interés.

Recibido el 18 de julio de 2019, aceptado el 15 de enero de 2020.

Correspondencia a: Dr. Nicolás Lorenzini CESFAM María Latife Saadi, Avenida Nelsón Pereira 2411. Rancagua, Chile. nlorenziniv@gmail.com

\section{Prueba diagnóstica de disfunción sudomotora en la detección precoz de la neuropatía diabética}

\author{
NICOLÁS LORENZINI ${ }^{1, \mathrm{a}}$, CLAUDIO DÍAZ ${ }^{1}$, TAMARA QUINTANA ${ }^{1, \mathrm{~b}}$
}

\section{Sudomotor dysfunction diagnostic test for early detection of diabetic neuropathy}

Background: Sudomotor dysfunction may appear in early stages of diabetic neuropathy. Aim: To evaluate the diagnostic capacity of the Neuropad test, based on the detection of sudomotor dysfunction, as an early indicator of diabetic neuropathy. Material and Methods: In Forty-two type 2 diabetic patients, the Neuropad test was compared with the $10 \mathrm{~g}$ monofilament test (proposed in the technical orientation of diabetic foot of the Ministry of Health of Chile), deep and thermal sensitivity. Results: The surface sensitivity assessed with a brush had a sensitivity and specificity of 18.8 and 100\% respectively when compared with the $10 \mathrm{~g}$ monofilament. When compared with the Neuropad, the figures were 9 and $100 \%$, respectively. Pain perception sensitivity and specificity were 13 and $100 \%$ respectively when compared with the $10 \mathrm{~g}$ monofilament. The figures were 6 and $100 \%$, when compared with the Neuropad. Thermal discrimination had a sensitivity and specificity of 88 and $33 \%$ respectively when compared with the 10 $g$ monofilament. The figures were 75 and $25 \%$ respectively when compared with the Neuropad. The deep sensitivity evaluated with a $128 \mathrm{~Hz}$ tuning fork had a sensitivity and specificity of 31 and 100\% respectively when compared with the $10 \mathrm{~g}$ monofilament. The figures were 16 and $31 \%$ respectively when compared with the Neuropad. The Neuropad had a sensitivity and specificity of 94 and 29\% respectively were compared with the $10 \mathrm{~g}$ monofilament. Conclusions: Neuropad had a good diagnostic yield for the early detection of sudomotor dysfunction.

(Rev Med Chile 2020; 148: 54-59)

Key words: Diabetic Neuropathies; Sensitivity and Specificity; Small Fiber Neuropathy; Sweating.

\section{L} a Diabetes Mellitus (DM) es un trastorno metabólico multisistémico, causado por múltiples factores que provocan un mal funcionamiento en la captación de insulina o de la secreción de ésta, provocando un estado de hiperglicemia crónica que finalmente lleva a complicaciones micro y macrovasculares.

En Chile, la Encuesta Nacional de Salud (ENS) del 2016-2017, estableció una prevalencia de $12,3 \%$ para la $\mathrm{DM}^{1}$. En nuestro país, se calcula que existen 1.372 .700 personas con DM, lo que representa $11 \%$ de la población adulta.
Aproximadamente $15 \%$ a $25 \%$ de los diabéticos desarrolla una úlcera en los pies a lo largo de su vida, la que puede evolucionar a una lesión grave y amputación. Según el registro de egresos hospitalarios del sector público de salud del año 2017, 67,9\% del total de las amputaciones de extremidad inferior son a causa de una úlcera de pie diabético (UPD).

El componente causal más frecuente de la UPD es la neuropatía periférica distal (NPD). La prevalencia de la neuropatía está entre 5,5 a $66 \%$ dependiendo del tipo población estudiada, 
la forma en que se realiza el estudio, el control metabólico y la duración de la $\mathrm{DM}^{2}$. Sin embargo, se estima que se pueden demostrar alteraciones electrofisiológicas en casi $100 \%$ de los diabéticos ${ }^{3}$.

El gold estándar para la evaluación de NPD son los estudios de electrodiagnóstico (electromiografía sensitiva y motora), que se considera la herramienta más sensible de diagnóstico. Son pruebas específicas y reproducibles, pero que requieren de servicios especializados, un elevado control y especificidad en las condiciones de aplicación, y expertos para su interpretación. Sin embargo, estos métodos no están al alcance de la atención primaria de salud.

Clínicamente NPD incluye un grupo de síntomas y signos clínicos con manifestaciones como parestesias, hiperestesia, ardor, alodinia, sensaciones eléctricas o punzantes.

La característica principal de la NPD es el daño precoz de las fibras nerviosas cortas, que representan el $70 \%$ de la inervación ${ }^{4}$, a su vez, estas se clasifican en $A \delta$ y C; las primeras, están ligeramente mielinizadas y se encargan de la percepción del frío y de la nocicepción cutánea. Las fibras $C$ no están mielinizadas y actúan como preganglionares y postganglionares y se encargan de la sensación térmica y el dolor, así como de funciones autónomas como el sudor ${ }^{5}$.

La evaluación de la NPD en la actualidad en Chile, se basa en pruebas que evalúan el estado de fibras nerviosas largas que sólo constituyen el $30 \%$ de los nervios periféricos ${ }^{6}$, los cuales están protegidos por una vaina de mielina, y se subdividen en $\alpha$ y $\beta$, siendo responsables del reflejo aquiliano, del tacto, de la sensibilidad vibratoria y la propiocepción?

La principal prueba sensorial utilizada de acuerdo a la recomendación técnica del Ministerio de Salud de Chile, es el monofilamento de $5,07 / 10 \mathrm{~g}^{8}$, para probar un punto de presión táctil, y determinar la presencia o ausencia de sensación protectora ${ }^{9}$.

Neuropad ${ }^{\circledR 7,10-13}$ se presenta como una alternativa diagnóstica que permite la detección precoz de la disfunción sudomotora, como indicador del daño las fibras nerviosas cortas, favoreciendo la detección temprana de una NPD.

\section{Objetivo}

Evaluar la sensibilidad y especificidad diagnóstica del Neuropad ${ }^{\circledR}$, frente al uso del monofi- lamento, para determinar cuál prueba presenta la mejor capacidad para la detección de la neuropatía diabética periférica,en pacientes de la Atención Primaria de Salud.

\section{Materiales y Métodos}

Se realizó un estudio de análisis de pruebas diagnósticas, en el que se comparó la sensibilidad y especificidad del Neuropad ${ }^{\circledR}$, versus el monofilamento para la detección de NPD en pacientes con DM tipo 2.

En un mismo paciente se realizaron ambas pruebas, con enmascaramiento para los investigadores, un investigador, realizó la evaluación recomendada por la orientación técnica para el manejo de pie diabético del Ministerio de Salud de Chile y el otro investigador, aplicó la prueba Neuropad ${ }^{\circledR}$. La información recopilada fue analizada por un tercer investigador, encargado del análisis estadístico.

Se realizó un muestreo aleatorio de pacientes diagnosticados con DM tipo 2 y sin diagnóstico previo de NPD, en control en el Programa Cardiovascular del Centro de Salud Familiar "María Latife Saadi", y se realizó un estudio de corte transversal.

Las variables analizadas fueron la sensibilidad superficial evaluada con el monofilamento. También se evaluó sensibilidad superficial con un pincel, capacidad de discriminar dolor y discriminar la variación térmica. La sensibilidad profunda se evaluó con el diapasón de $128 \mathrm{~Hz}^{2}$.

Estas variables fueron comparadas en su especificidad y sensibilidad para el monofilamento y para el Neuropad ${ }^{\circledR}$ es una prueba a base de un indicador visual, que utiliza un cambio de color para definir la integridad de la inervación colinérgica simpática de la piel. El Cloruro de cobalto que tiene el dispositivo cambia de color azul a rosa en un tiempo máximo de $10 \mathrm{~min}$. Si cambia de color la prueba se considera como negativa para NPD ${ }^{10-}$ ${ }^{14}$. Esta prueba ya ha sido aceptada como una de las pruebas de valoración de neuropatía diabética por el Panel de Consenso de Toronto ${ }^{15}$.

La aplicación se deber realizar con el paciente en una habitación con a una temperatura constante, con los pies descalzos, al menos 5 min antes del examen. Se aplica el parche en un área sin hiperqueratosis en la zona plantar, entre la primera 


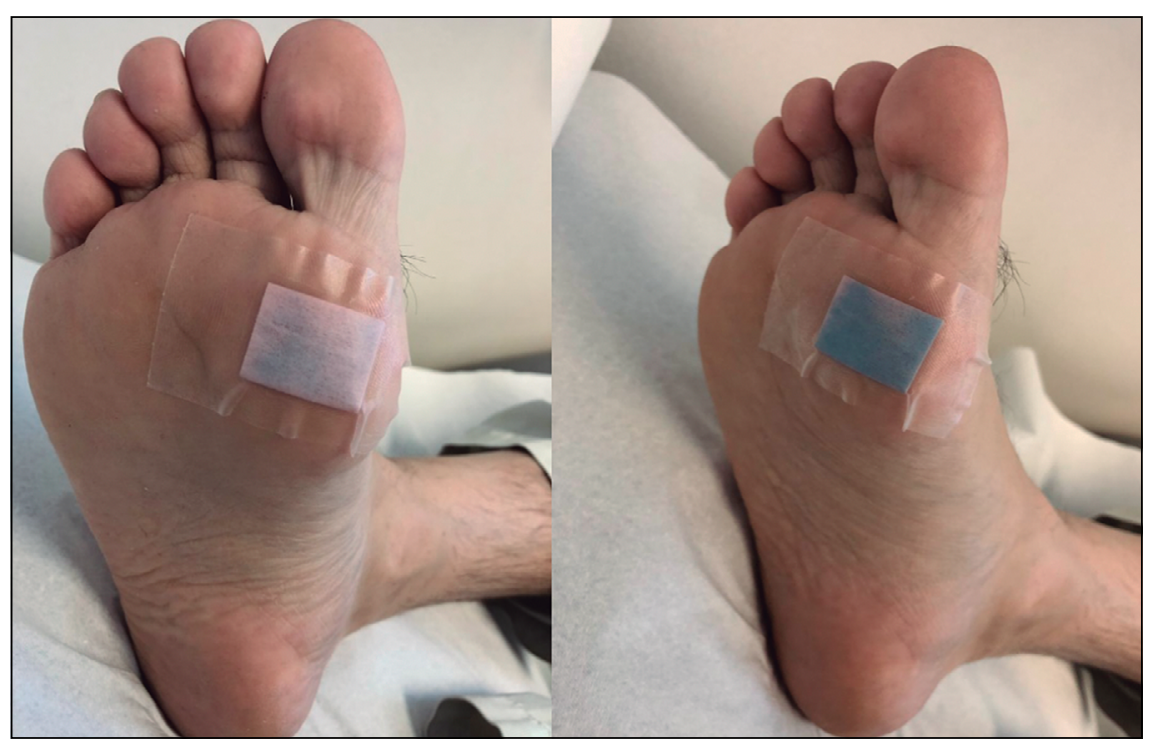

Figura 1. Aplicación correcta del Neuropad ${ }^{\circledR}$. Fotografía izquierda: tiempo 0 de aplicación. Fotografía derecha: tiempo $10 \mathrm{~min}$ de aplicación. y la segunda cabeza metatarsal de forma bilateral. Se considera una respuesta normal, el cambio completo de color de azul a rosa a los $10 \mathrm{~min}$ de su aplicación (Figura 1).

La muestra consta de 40 pacientes diabéticos tipo 2, elegidos de forma aleatoria, (16 mujeres y 24 hombres. Los pacientes fueron reclutados acorde a los criterios de inclusión a medida que ingresaban al programa de salud cardiovascular del centro de salud familiar, entre los meses de enero y mayo de 2019.

El estudio fue realizado en el Centro de Salud Familiar "María Latife Saadi", de la comuna de Rancagua, siendo aprobado por el Comité Ética de la Corporación Municipal de Rancagua. Además, todos los participantes de este estudio firmaron el Consentimiento Informado, aprobado por este comité, en señal de aceptación.

Los criterios de inclusión fueron:

- Diagnóstico establecido de DM tipo 2.

- Sin diagnóstico previo de neuropatía diabética.

- Edad entre 20 y 80 años.

- Ser un paciente habitual en control en el Programa Cardiovascular del Centro de Salud.

Como criterios de exclusión se definieron:

- Paciente con diagnóstico previo de neuropatía de cualquier otra etiología.

- Pacientes con síndrome nefrótico.
- Alcoholismo.

- Cirrosis o enfermedades hepáticas crónicas.

- Enfermedad renal.

- Pacientes sometidos a quimioterapia o que se encuentren actualmente en quimioterapia.

- Déficit de vitamina B12 o anemia.

- Paciente portador de cualquier enfermedad oncológica.

- Paciente portador de virus de inmunodeficiencia humana.

- Pacientes con diagnóstico de vasculitis o neuropatías congénitas.

- Pacientes que hayan sufrido infarto agudo de miocardio en los últimos 6 meses previos a ingresar al estudio.

- Pacientes con diagnóstico de hipotiroidismo no controlado o tirotoxicosis.

- Pacientes con secuelas de amputaciones previas.

- Pacientes portadores de enfermedad vertebral.

- Pacientes que en el control cardiovascular hayan evidenciado alguna complicación aguda de la DM manifestada por episodios de hipoglicemia o hiperglicemia.

El tamaño de la muestra fue de 40 pacientes.

\section{Análisis estadístico}

El análisis estadístico se realizó con el programa IBM SPSS Statistics Subscription. El análisis fue 
realizado por la prueba de $\mathrm{CHI}$ cuadrado (usando tablas de contingencia de $2 \times 2$ ), para las variables cualitativas dicotómicas.

Se determinó la Sensibilidad (S), Especificidad (E), Valor Predictivo Positivo (VPP), Valor Predictivo Negativo (VPN), Proporción De Falsos Positivos (PFP) y de Falsos Negativos (PFN).

\section{Resultados}

El examen de diagnóstico de NPD, recomendado en la orientación técnica del Ministerio de Salud de Chile (MINSAL) del año 2018, considera la utilización de 2 instrumentos cualitativos sensoriales (diapasón $128 \mathrm{~Hz}$ y MF), como criterio de referencia de NPD, no siendo utilizados otros instrumentos como el pincel, la evaluación del dolor (clip, pinprick ${ }^{\circledR}$, neurotip ${ }^{\circledR}$ ) o la barra térmica.
Se utilizó como estándar el MF, y se comparó con el pincel, dolor, sensibilidad térmica y diapasón en términos de su sensibilidad y especificidad, valores predictivos y proporciones; para la detección de NPD (Tabla 1).

Se realizaron los mismos cálculos para la aplicación de Neuropad ${ }^{\circledR}$ (Tabla 2) y se realizó la comparación para la detección de NPD con el MF y MF asociado a diapasón frente al Neuropad ${ }^{\circledR}$. (Tabla 3).

$\mathrm{Al}$ utilizar MF como instrumento para determinar NPD, se encontró $40 \%$ de individuos con un resultado positivo, mientras que para el Neuro$\operatorname{pad}^{\circledR} 80 \%$ de los individuos resultaron positivos.

Para la de evaluación sensorial superficial, destaca la prueba de discriminación térmica que presentó una elevada sensibilidad para ambas pruebas, $87,5 \%$ para MF y $75 \%$ para Neuropad ${ }^{\circledR}$. Las pruebas sensoriales superficiales pincel, discri-

Tabla 1. Pruebas diagnósticas versus monofilamento

\begin{tabular}{|lcccccccc|}
\hline & S (\%) & IC (\%) & E (\%) & IC (\%) & VPP (\%) & VPN (\%) & PFP (\%) & PFN (\%) \\
\hline Pincel vs MF & 18,8 & 6,6 a 43 & 100 & 86,2 a 100 & 100 & 64,9 & 0 & 81,3 \\
\hline Dolor vs MF & 12,5 & 3,5 a 36 & 100 & 86,2 a 100 & 100 & 63,4 & 0 & 87,5 \\
\hline Térmica vs MF & 87,5 & 64,0 a 96,5 & 33,3 & 18,0 a 53,3 & 46,7 & 80 & 66,7 & 12,5 \\
\hline Diapason vs MF & 31,3 & 14,2 a 55,6 & 100 & 86,2 a 100 & 100 & 68,6 & 0 & 68,8 \\
\hline
\end{tabular}

Monofilamento (MF), Intervalo de confianza (IC). Sensibilidad (S), Especificidad (E), Valor Predictivo Positivo (VPP), Valor Predictivo Negativo (VPN), Proporción de Falsos Positivos (PFP) y de Falsos Negativos (PFN).

Tabla 2. Pruebas diagnósticas versus Neuropad ${ }^{\circledR}$

\begin{tabular}{|lcccccccc|}
\hline & S (\%) & IC (\%) & E (\%) & IC (\%) & VPP (\%) & VPN (\%) & PFP (\%) & PFN (\%) \\
\hline Pincel vs Neuropad $^{\circledR}$ & 9,4 & 3,2 a 24,2 & 100 & 67,6 a 100 & 100 & 21,6 & 0 & 90,6 \\
Dolor vs Neuropad $^{\circledR}$ & 6,3 & 1,7, a 20,1 & 100 & 67,6 a 100 & 100 & 21,1 & 0 & 93,8 \\
\hline Termica vs Neuropad $^{\circledR}$ & 75 & 57,9 a 86,7 & 25 & 7,1 a 59,1 & 80 & 20 & 75 & 25 \\
\hline Diapason vs Neuropad $^{\circledR}$ & 15,6 & 6,9 a 31,8 & 100 & 67,6 a 100 & 100 & 22,9 & 0 & 84,4 \\
\hline
\end{tabular}

Intervalo de confianza (IC). Sensibilidad (S), Especificidad (E), Valor Predictivo Positivo (VPP), Valor Predictivo Negativo (VPN), Proporción de Falsos Positivos (PFP) y de Falsos Negativos (PFN).

Tabla 3. Neuropad ${ }^{\circledR}$ versus prueba diagnostica monofilamento y monofilamento-diapason $128 \mathrm{HZ}$

\begin{tabular}{|c|c|c|c|c|c|c|c|c|}
\hline & S (\%) & IC (\%) & E (\%) & IC (\%) & VPP (\%) & VPN (\%) & PFP (\%) & PFN (\%) \\
\hline Neuropad $^{\circledR}$ vs MF & 93,6 & 71,7 a 98,9 & 29,2 & 14,9 a 49,2 & 46,9 & 87,5 & 70,8 & 6,3 \\
\hline Neuropad ${ }^{\circledR}$ vs MF-Diapasón & 93,8 & 71,7 a 98,9 & 29,2 & 14,9 a 49,2 & 46,9 & 87,5 & 70,8 & 6,3 \\
\hline
\end{tabular}

Monofilamento (MF), Intervalo de confianza (IC). Sensibilidad (S), Especificidad (E), Valor Predictivo Positivo (VPP), Valor Predictivo Negativo (VPN), Proporción de Falsos Positivos (PFP) y de Falsos Negativos (PFN). 
minación al dolor y de sensibilidad profunda con diapasón tienen $100 \%$ de especificidad, pero una baja sensibilidad para ambas pruebas.

Al contrastar el Neuropad ${ }^{\circledR}$ con el MF y MF asociado a diapasón de $128 \mathrm{~Hz}$ la sensibilidad es de $93,2 \%$ y una especificidad de $29, \%$. Pero al realizar el cálculo de la proporción de falsos positivos, encontramos 70,8\% de casos en los cuales la prueba de Neuropad ${ }^{\circledR}$ sigue siendo positiva, pero el MF detecta como negativa para de NPD.

\section{Discusión}

En base a los resultados de este estudio, la validez diagnóstica del Neuropad ${ }^{\circledR}$ para la detección de neuropatía periférica radica en su elevada sensibilidad. Mientras que la baja especificidad del Neuropad ${ }^{\circledR}$ frente al MF, reside en la fisiopatología de la NPD, que afecta primero a las fibras nerviosas cortas, lo que lleva a la presencia de anhidrosis por la denervación de las glándulas sudoríparas afectando la función sudomotora, la cual es detectada por el Neuropad ${ }^{\circledR}$ y no por el MF.

Se comparó el resultado de este estudio con 5 estudios previos ${ }^{7,10-13}$ obteniéndose resultados similares en términos de sensibilidad. En estos estudios se utilizó Neuropad ${ }^{\circledR}$ para la evaluación NPD en comparación a métodos tradicionales, con un total de 967 individuos. La media para la sensibilidad fue de $89,4 \% \pm 12,2$ y su especificidad de $43,4 \% \pm 15,1$. En uno de estos estudios se comparó Neuropad ${ }^{\circledR}$ frente a la electromiografía ${ }^{7}$ en donde se obtuvo una sensibilidad y especificidad del $95,2 \%$ y $67,2 \%$ respectivamente.

Por otra parte, tener una prueba alterada de Neuropad ${ }^{\circledR}$, según meta-análisis publicado en 2011, supone un riesgo casi diez veces mayor OR $[9,54(7,10-12,83)]$ de padecer neuropatía periférica $^{16}$.

A la fecha el MF ha sido el método de elección para el diagnóstico de neuropatía diabética, siendo su mayor ventaja su mínimo costo. Sin embargo, presenta limitaciones como ser operador dependiente y los cuidados que se deben tener con el instrumento.

En 2010 una revisión del tema realizada por Tan $\mathrm{LS}^{8}$ señala que no hay consenso sobre el protocolo de uso del monofilamento respecto de la ubicación y el número de sitios probados, lo que afecta la afecta a la sensibilidad y especificidad del instrumento para la detección de NPD. En el año 2017 se realizó un meta análisis ${ }^{17}$ en el cual sensibilidad y especificidad de las pruebas de monofilamento para detectar la neuropatía periférica diabética fueron 0,53 (intervalo de confianza (IC) del $95 \%$ : 0,32 a 0,74 ) y 0,88 (IC del $95 \%$ : 0,78 a 0,94), respectivamente. Lo cual demostró que el monofilamento tiene una sensibilidad limitada para detectar neuropatía periférica diabética.

Neuropad ${ }^{\circledR}$ es una prueba que presentó una alta sensibilidad en la detección precoz de la NPD. Otro aspecto es que pacientes con exploración neurológica normal con MF pueden presentar una prueba alterada de Neuropad ${ }^{\circledR}$, lo que sugiere que la afectación sudomotora ocurre en las primeras fases NPD, lo que permite considerar la posibilidad de utilizar esta prueba en la detección temprana de la misma.

A diferencia del MF, Neuropad ${ }^{\circledR}$ no es operador dependiente, ni requiere de personal especializado, lo que favorece al autoexamen. La limitación Neuropad ${ }^{\circledR}$ es la posible alteración de la sudoración de forma fisiológica, que se produce en los pacientes de edad avanzada.

En resumen tanto este estudio con la literatura existente avalan el uso del Neuropad ${ }^{\circledR}$ como una prueba para la detección precoz de NPD, y confirman que el uso del MF presenta limitaciones para la detección temprana de la NPD.

La conclusión de esta investigación sería recomendar la incorporación de Neuropad ${ }^{\circledR}$, de forma rutinaria en la práctica clínica como herramienta de cribado, en todo paciente con un diagnóstico inicial de DM, como también su utilización en combinación con los métodos recomendados en la orientación técnica del MINSAL. De esta manera se lograría una detección precoz de individuos con una NPD, lo que permitiría tomar medidas preventivas con el objetivo de disminuir el número de futuras amputaciones.

\section{Referencias}

1. Subsecretaria de Salud Pública de Chile, Departamento de Epidemiología. Encuesta Nacional de Salud 20162017. Primeros Resultados. Disponible en: http://web. minsal.cl/wp-content/uploads/2017/11/ENS-201617_PRIMEROS-RESULTADOS.pdf (acceso el 22 de enero de 2020).

2. International Diabetes Federation: Clinical Practice 
Recommendations on the Diabetic Foot: A guide for heath care professionals: Internationa Diabetes Federation, 2017 [Internet] [citado el 30 de octubre de 2017]. Disponible en: https://www.idf.org/e-library/guidelines/119-idf-clinical-practice-recommendations-on-diabetic-foot-2017.html.

3. Klein R, Klein BE, Moss SE. Relation of glycemic control to diabetic microvascular complications in diabetes mellitus. Annals of Internal Medicine 1996; 124: 90-5.

4. Løseth S, Stålberg E, Jorde R, Mellgren SI. Early diabetic neuropathy: thermal thresholds and intraepidermal nerve fibre density in patients with normal nerve conduction studies. J Neurol 2008; 255 (8): 1197-202.

5. Olaleye D, Perkins BA, Bril V. Evaluation of three screening tests and a risk assessment model for diagnosing peripheral neuropathy in the diabetes clinic. J Peripheral Nervous System 2002; 7 (2): 137.

6. Quattrini C, Jeziorska M, Malik RA. Small fiber neuropathy in diabetes: clinical consequence and assessment. Int J Low Extrem Wounds 2004; 3 (1): 16-21.

7. Papanas N, Giassakis G, Papatheodorou K, Papazoglou D, Monastiriotis C, Christakidis D, et al. Sensitivity and specificity of a new indicator test (Neuropad) for the diagnosis of peripheral neuropathy in type 2 diabetes patients: a comparison with clinical examination and nerve conduction study. J Diabetes Complications 2007; 21 (6): 353-8.

8. Tan LS. The clinical use of the $10 \mathrm{~g}$ monofilament and its limitations: a review. Diabetes Res Clin Pract 2010; 90 (1): 1-7.

9. Orientación Técnica Manejo Integral Del Pie Diabético 2018. Subsecretaría De Salud Pública División De Prevención Y Control De Enfermedades Departamento De Enfermedades No Transmisibles. Ministerio De Salud De Chile. Disponible en: https://www.capacitacionesonline.com/blog/wp-content/uploads/2019/07/
Orientación-Técnica-Manejo-integral-del-pie-diabético. (Acceso el 22 de enero de 2020).

10. Mendivil CO, Kattah W, Orduz A, Tique C, Cárdenas JL, Patiño JE. Neuropad for the detection of cardiovascular autonomic neuropathy in patients with type 2 diabetes. J Diabetes Complications 2016; 30 (1): 93-8.

11. Sanz-Corbalán I, Lázaro-Martínez JL, García-Morales E, Molines-Barroso R, Álvaro-Afonso F, García-Álvarez Y. Advantages of early diagnosis of diabetic neuropathy in the prevention of diabetic foot ulcers. Diabetes Res Clin Pract 2018; 146: 148-54.

12. Spallone V, Morganti R, Siampli M, Fedele T, D'Amato C, Cacciotti L. Neuropad as a diagnostic tool for diabetic autonomic and sensorimotor neuropathy. Diabet Med. 2009; 26 (7): 686-92.

13. Tentolouris N, Voulgari C, Liatis S, Kokkinos A, Eleftheriadou I, Makrilakis $\mathrm{K}$, et al. Moisture status of the skin of the feet assessed by the visual test neuropad correlates with foot ulceration in diabetes. Diabetes Care 2010; 33 (5): 1112-4.

14. Papanas N, Ziegler D. New diagnostic tests for diabetic distal symmetric polyneuropathy. J Diabetes Complications 2011; 25 (1): 44-51.

15. Malik RA, Veves A, Tesfaye S, Smith G, Cameron N, Zochodne D, et al. Small fibre neuropathy: role in the diagnosis of diabetic sensorimotor polyneuropathy. Diabetes Metab Res Rev 2011; 27 (7): 678-84.

16. Tentolouris N, Kokkinos A, Diakoumopoulou E, Eleftheriadou I, Alexiadou K. The association between Neuropad testing with peripheral neuropathy in diabetes: a meta-analysis. Diabetologia 2011; 54: A1154. .

17. Wang F, Zhang J, Yu J, Liu S, Zhang R, Ma X, et al. Diagnostic Accuracy of Monofilament Tests for Detecting Diabetic Peripheral Neuropathy: A Systematic Review and Meta-Analysis. J Diabetes Res 2017; 2017: 8787261. doi:10.1155/2017/8787261. 